Revue des patrimoines

\title{
Archéologie, architecture et ébénisterie : les meubles de la villa Kérylos à Beaulieu-sur-Mer
}

Françoise Reynier

\section{(2) OpenEdition}

Journals

Édition électronique

URL : http://journals.openedition.org/insitu/9376

DOI : $10.4000 /$ insitu.9376

ISSN : 1630-7305

Éditeur

Ministère de la culture

Référence électronique

Françoise Reynier, «Archéologie, architecture et ébénisterie : les meubles de la villa Kérylos à

Beaulieu-sur-Mer », In Situ [En ligne], 6 | 2005, mis en ligne le 01 septembre 2005, consulté le 30 avril 2019. URL : http://journals.openedition.org/insitu/9376 ; DOI : 10.4000/insitu.9376

Ce document a été généré automatiquement le 30 avril 2019.

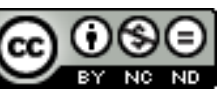

In Situ Revues des patrimoines est mis à disposition selon les termes de la licence Creative Commons Attribution - Pas d'Utilisation Commerciale - Pas de Modification 4.0 International. 


\title{
Archéologie, architecture et ébénisterie : les meubles de la villa Kérylos à Beaulieu-sur-Mer
}

\author{
Françoise Reynier
}

1 La villa Kérylos fut construite à Beaulieu-sur-Mer, de 1902 à 1908, par Emmanuel Pontremoli, à la demande de Théodore Reinach ${ }^{1}$, érudit passionné par l'Antiquité grecque dont il connaissait parfaitement les diverses facettes. Outre ses talents d'architecte, Pontremoli possédait une solide expérience d'archéologue, étayée par une grande capacité d'analyse et de réflexion sur la genèse des monuments qu'il avait reconstitués à partir des vestiges de Pergame ou de Didymes. Lorsqu'ils se rencontrèrent en 1900, Reinach venait d'acquérir un promontoire rocheux à l'extrémité de la baie des Fourmis, dont la ressemblance avec la Grèce lui inspira la surprenante commande d'une maison à l'image de celles mises au jour par les récentes fouilles de Délos. Leur connaissance approfondie de l'art et de la culture grecque antiques permit aux deux hommes de donner naissance à une demeure exceptionnelle (fig. $\mathbf{n}^{\circ} \mathbf{1}$ ). L'architecte dessina également la totalité de la décoration intérieure et du mobilier, y compris la vaisselle et l'argenterie ${ }^{2}$, après en avoir choisi les thèmes ou les motifs avec le commanditaire. Il ne s'agissait pas alors de créer un musée, mais de construire une maison confortable, équipée de l'électricité, de l'eau courante et d'un chauffage central à air pulsé par le sol. Pontremoli réussit la prouesse d'intégrer et de dissimuler parfaitement tous ces anachronismes dans un cadre « antique ». 
Figure 1

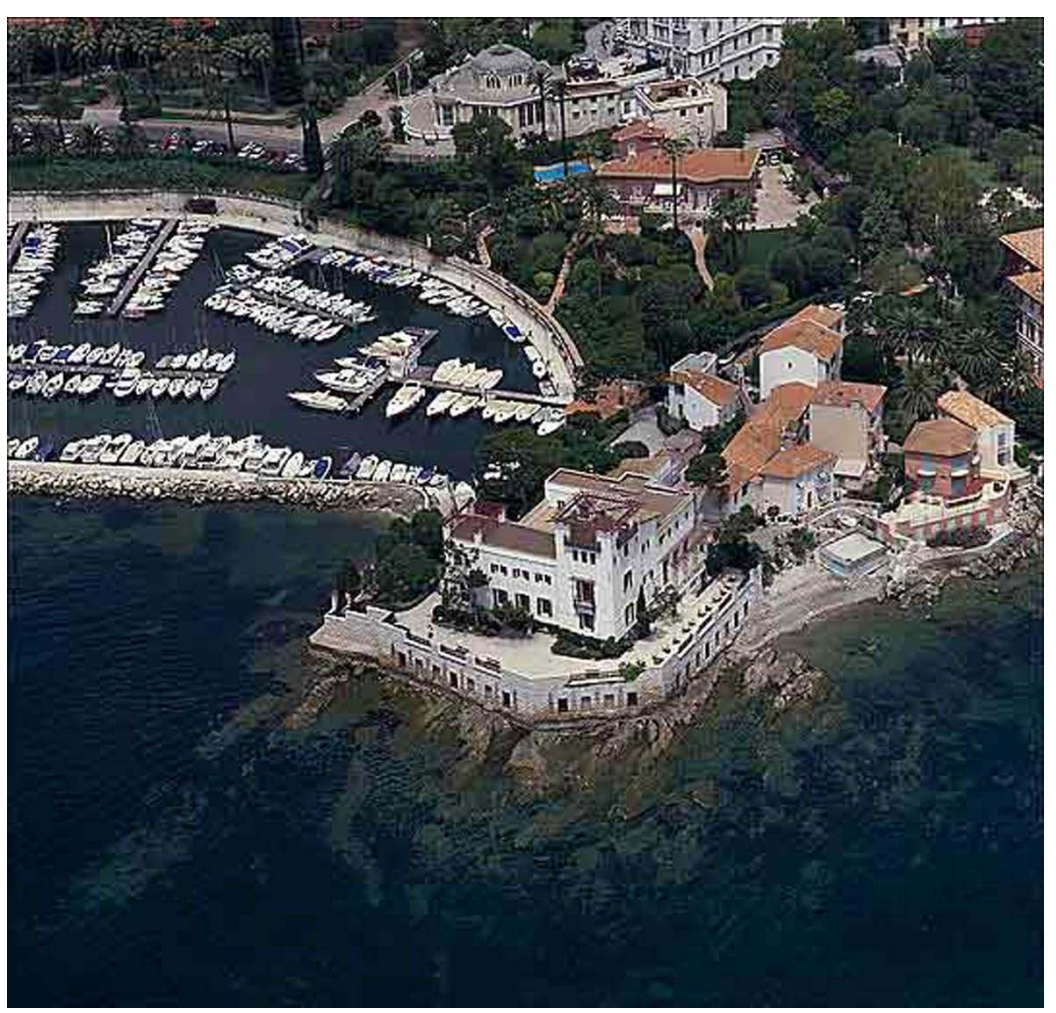

Beaulieu-sur-Mer. Villa Kérylos : vue aérienne, prise du sud-est.

Phot. Inv. M. Heller-G. Roucaute (c) Inventaire général, ADAGP, 1998.

2 La topographie ne permet pas de doter la villa du plan carré classique; trois ailes entourent les côtés nord, est et sud du péristyle et la quatrième aile est accolée perpendiculairement au côté nord. Toutes les pièces de réception ouvrent à la fois sur le péristyle et sur le jardin. Les deux salons et la salle à manger sont disposés en enfilade sur le côté sud. A l'est, un vestibule distribue la vaste bibliothèque, la salle à manger et l'escalier principal, seul à desservir tous les niveaux. L'aile nord abrite une grande salle de bains d'apparat et deux petites chambres destinées aux invités.

3 Au premier étage, l'aile sud est consacrée aux appartements de Théodore Reinach et de son épouse. Les deux chambres sont séparées par leurs salles de bains, une cabine de douche et un salon de repos. Un autre appartement pour les invités, avec salle de bains identique aux précédentes, ouvre sur le côté nord du péristyle. Le second étage occupe un espace nettement plus réduit, celui de la «tour» et comprenait deux chambres avec cabinet de toilette. Enfin, une grande terrasse couverte par une pergola couronne l'ensemble et permet d'apprécier un somptueux panorama.

4 Toutes ces pièces sont desservies par un système complexe de couloirs, d'escaliers et de vestibules permettant de dissocier les espaces de service de ceux réservés aux « maitres ", comme dans les grandes demeures bourgeoises de l'époque. 


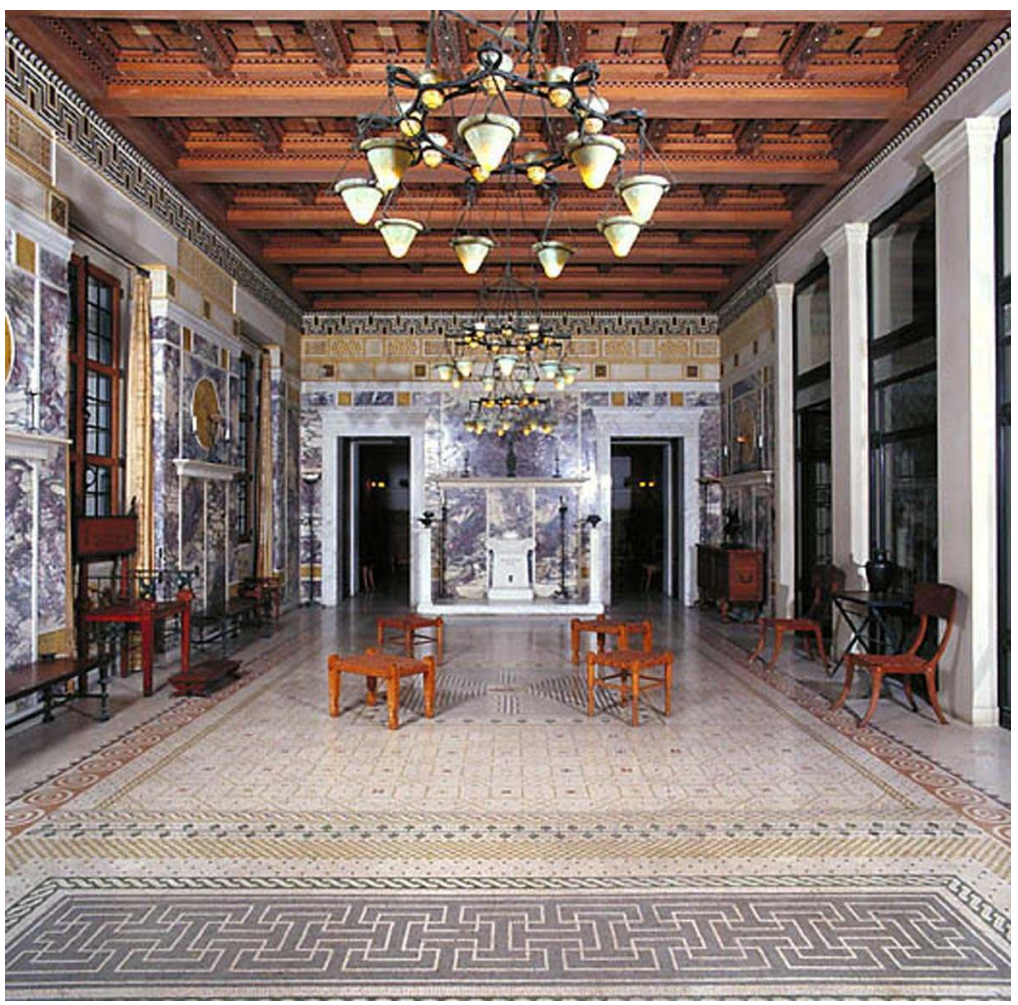

La partie ouest du grand salon.

Phot. Inv. M. Heller-G. Roucaute (c) Inventaire général, ADAGP, 1998.

Les sols sont revêtus de mosaïque, les murs sont couverts de fresques, plus ou moins ornementées, certains portent des stucs et les plafonds aux poutres apparentes sont peints (fig. $\mathbf{n}^{\circ} 2$ ). L'ensemble du décor et du mobilier dessinés par Pontremoli, dont une centaine de luminaires électriques, est directement inspiré de l'Antiquité, pas seulement grecque mais aussi romaine et, plus rarement, égyptienne. De nombreuses copies d'objets et de meubles en bronze conservés au musée archéologique de Naples le complètent. L'abondance et le raffinement des œuvres réalisées dans les ateliers de l'ébéniste parisien Louis-François Bettenfeld ${ }^{3}$ parachèvent le caractère luxueux de la demeure et en font une véritable résidence.

Le nombre de meubles meublants est impressionnant: environ deux cent-quatre-vingt ! Les pièces de séjour et les cinq chambres principales sont dotées chacune d'un ensemble en bois exotique décoré d'incrustations. Chaque chambre est garnie au minimum d'un lit, d'une table de nuit, d'une table avec une chaise, parfois de deux fauteuils, et d'une ou deux armoires appelées "coffres à vêtements" par Pontremoli (fig. $\mathbf{n}^{\circ}$ 3). Les petites chambres du rez-de-chaussée possédaient un équipement analogue, en bois peint de couleur bleue ou marron, rehaussée de blanc et d'ocre ${ }^{4}$. Outre les installations sanitaires, les salles de bains comportent une table de toilette avec chaise assortie, des coffres à linge et des tabourets. Les pièces de service et les cinq chambres des domestiques avaient également leur ameublement néo-grec, en chêne, dont une petite partie a été conservée. 
Figure 3

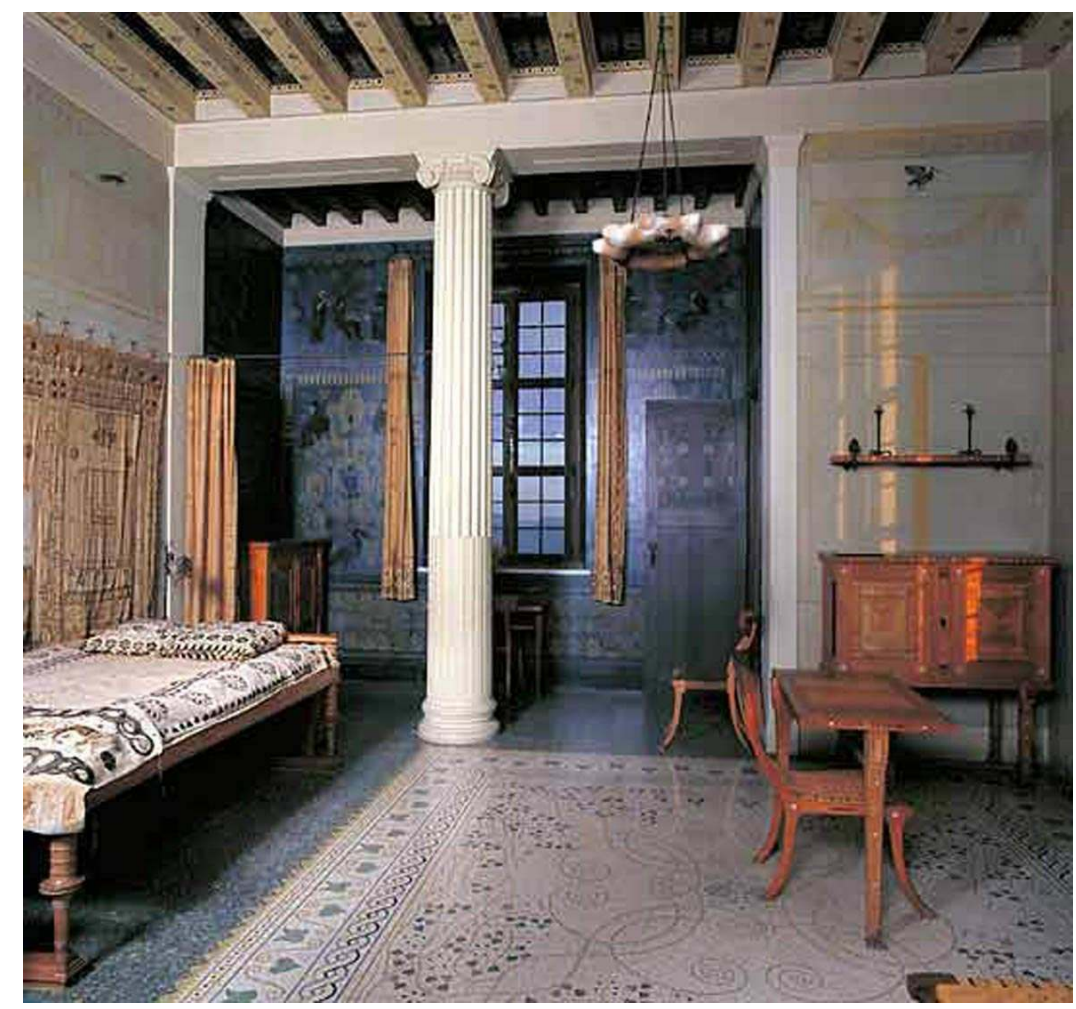

La chambre de Madame.

Phot. Inv. M. Heller-G. Roucaute (c) Inventaire général, ADAGP, 1998.

7 Malgré tout l'intérêt qu'ils présentent, les meubles de la villa n'ont pas encore fait l'objet d'une étude complète, ni de publication à part entière, contrairement aux mosaïques, aux fresques, aux stucs et aux bronzes qui ont été déjà largement commentés et sur lesquels nous ne reviendrons pas.

\section{Un mobilier « antique » et un confort « moderne »}

Les vases antiques à figures rouges ou noires présentent souvent des scènes d'intérieur dans lesquelles sont assez nettement visibles les sièges et les lits, les tables et les coffres qui constituent alors l'essentiel de l'ameublement. Les formes sont assez peu variées : les pieds et les montants des chaises sont très incurvés, presque toutes les tables ont trois pieds, souvent terminés en patte de lion (fig. $\mathbf{n}^{\circ}$ 4), les lits ont des pieds tournés avec des parties élargies en disque ou des pieds quadrangulaires décorés d'incrustations. Les coffres ont généralement de petits pieds ou sont posés sur des patins. 
Figure 4

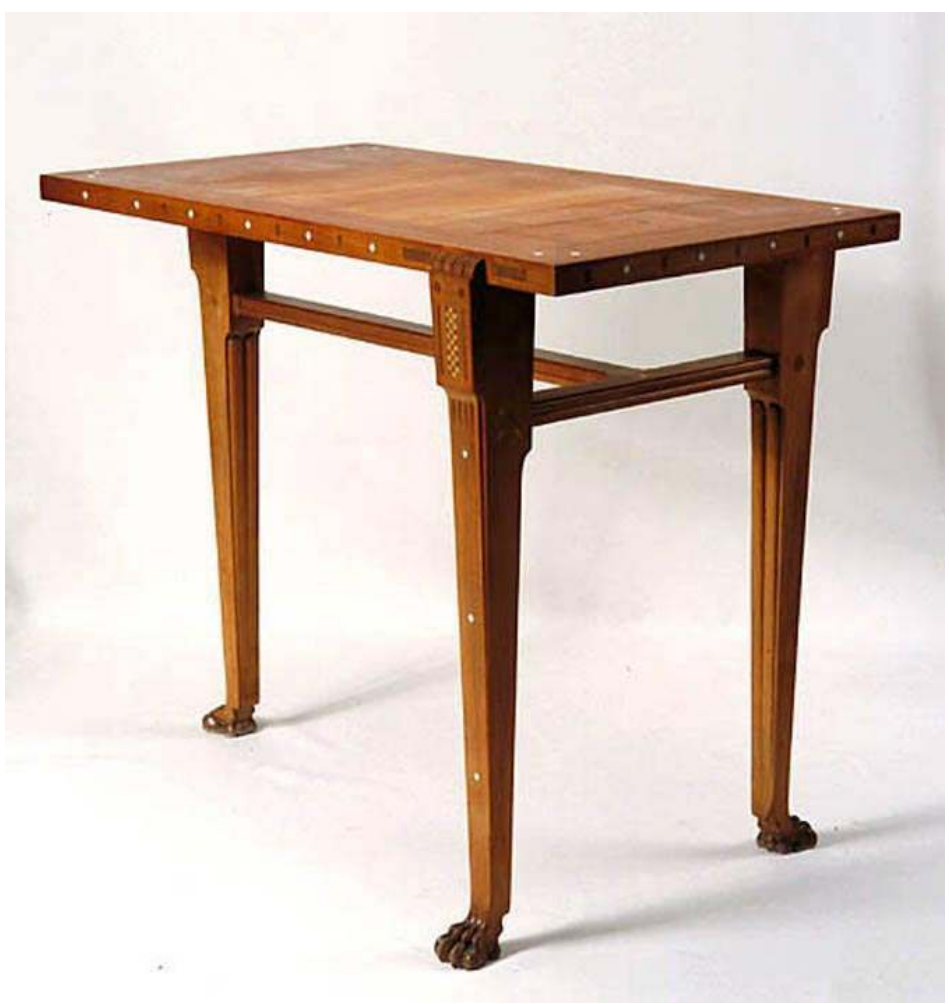

La table de la chambre de Madame.

Phot. Inv. M. Heller-G. Roucaute ( ) Inventaire général, ADAGP, 1998.

9 La plupart des meubles créés par Pontremoli présentent ces caractéristiques formelles, tout en étant adaptés à un confort qui n'existait pas dans l'Antiquité. Les salles de bains de Madame et de Monsieur comportent chacune un faux coffre qui contient un bidet, placé sur un fond coulissant et accessible grâce à un discret battant latéral (fig. $\mathbf{n}^{\circ}$ 5) (fig. $\mathrm{n}^{\circ}$ 6) 
Figure 5

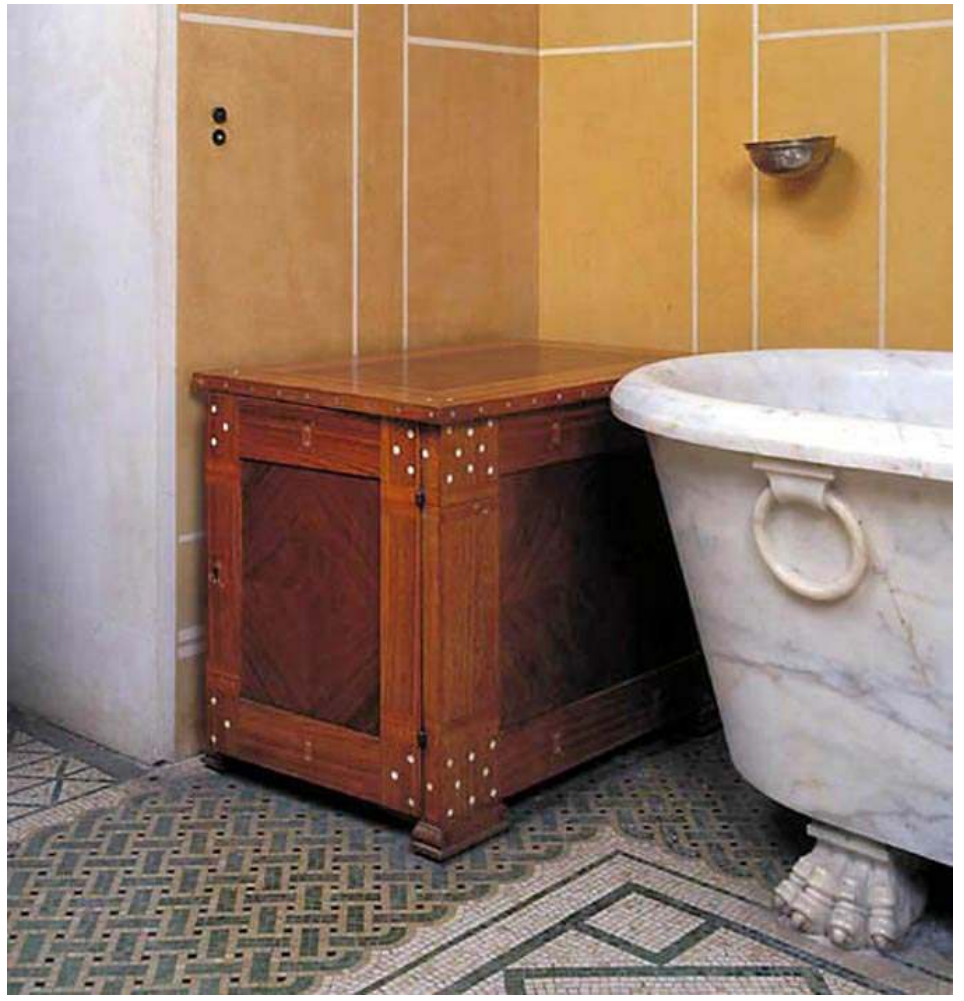

Le bidet de la salle de bains de Madame dans son meuble de rangement en forme de coffre, fermé. Phot. Inv. M. Heller-G. Roucaute (c) Inventaire général, ADAGP, 1998. 
Figure 6

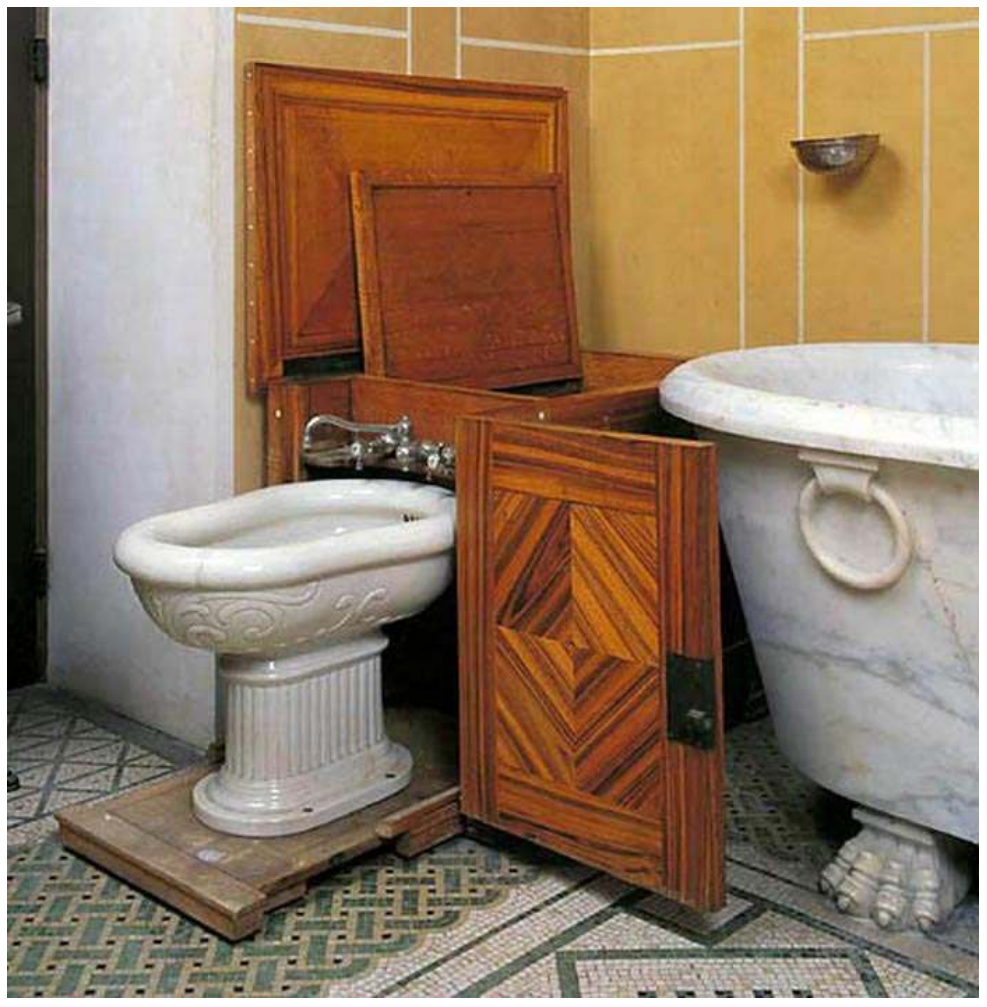

Le bidet de la salle de bains de Madame, installé.

Phot. Inv. M. Heller-G. Roucaute ( ) Inventaire général, ADAGP, 1998.

L'apparence d'un coffre est donnée à certains meubles de rangement, comme les buffets du grand salon, en encastrant astucieusement les battants dans le bâti, en réduisant les garnitures métalliques tout en ajoutant de grosses poignées tombantes en bronze sur les côtés et en simulant un piétement indépendant (fig. $\mathbf{n}^{\circ} 7$ ). 
Figure 7

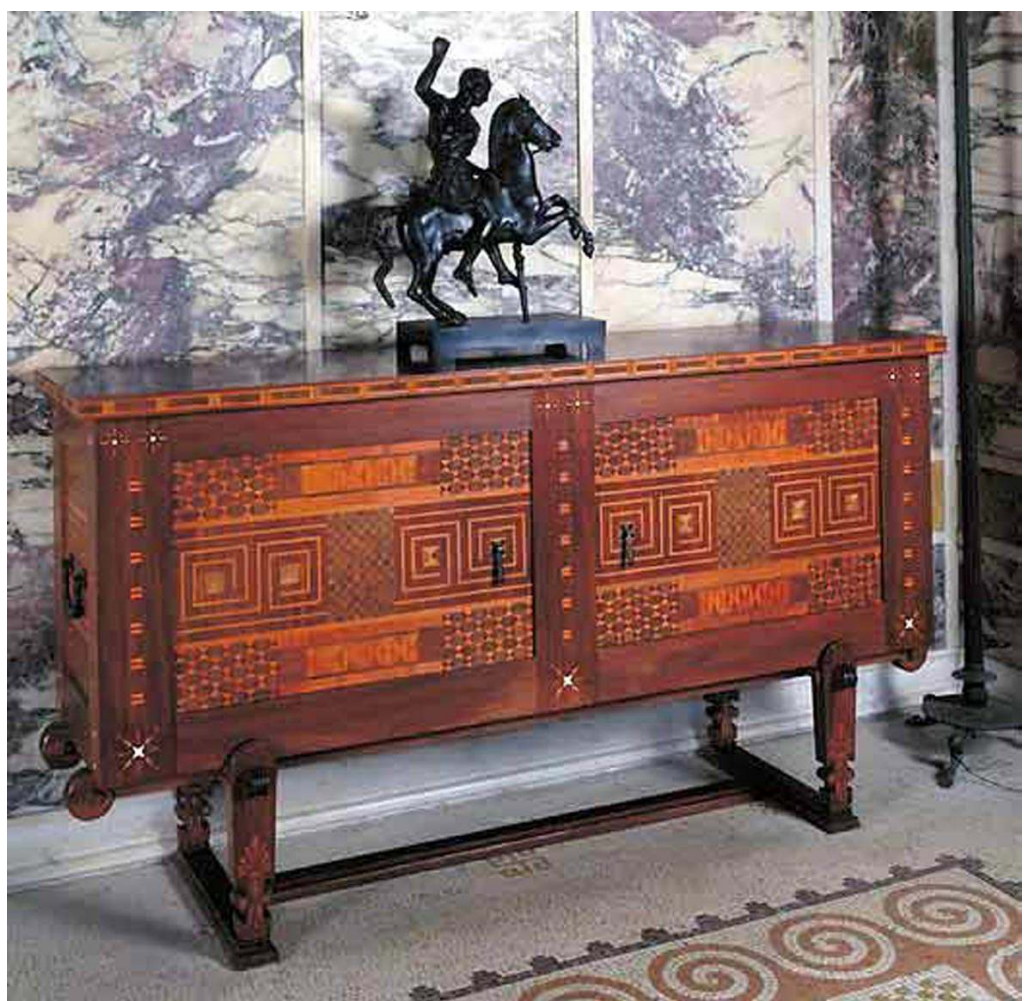

Un des deux buffets du grand salon, en cœur vert incrusté de cinq autres bois.

Phot. Inv. M. Heller-G. Roucaute (c) Inventaire général, ADAGP, 1998.

11 Un exemple de dissimulation presque parfaite est le petit « coffre » clouté de bronze de la bibliothèque, qui a en réalité deux battants et un tiroir en façade, un dessus fixe et qui est donc une armoire, selon la terminologie de l'Inventaire général (fig. $\mathbf{n}^{\circ} \mathbf{8}$ ). Mais, sur les autres buffets et armoires, les battants et les tiroirs restent bien visibles. 
Figure 8

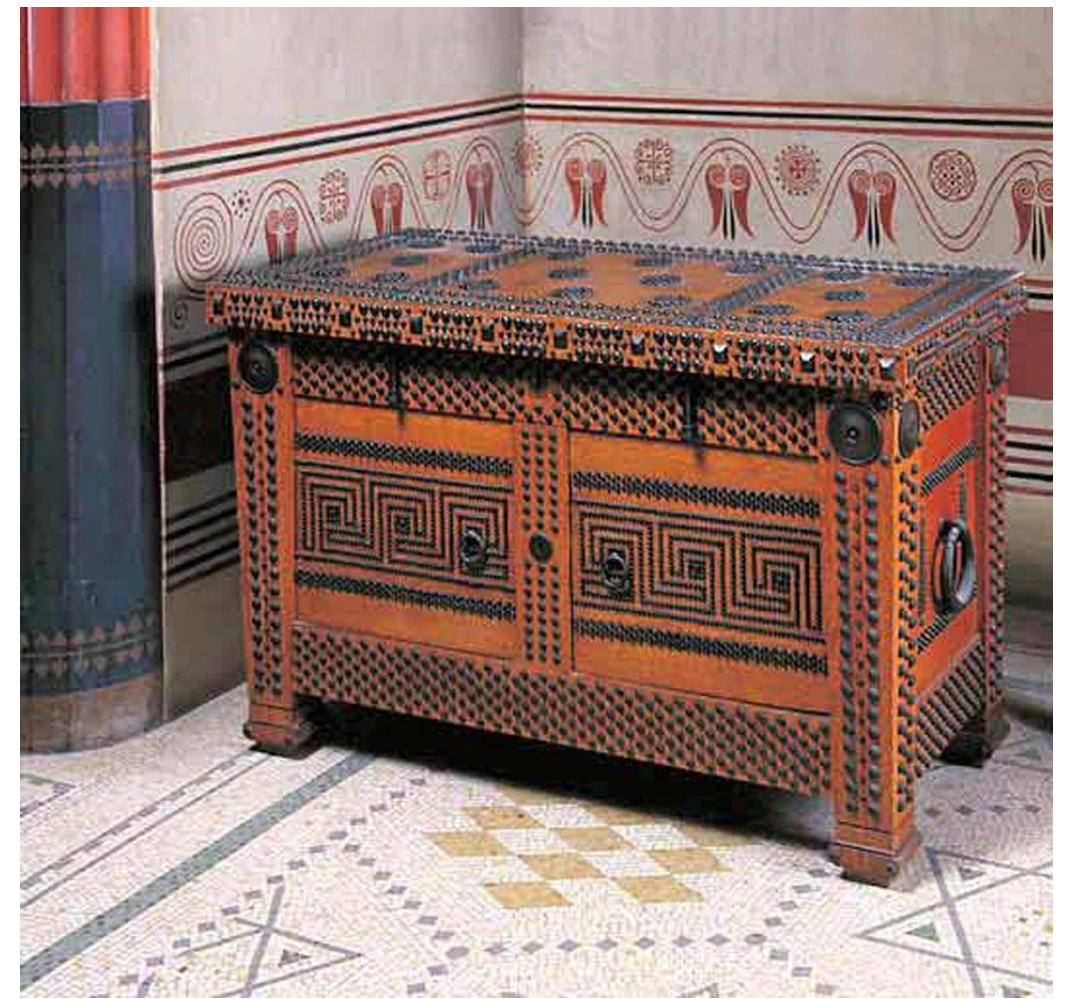

L'armoire à l'apparence de coffre, dans la bibliothèque.

Phot. Inv. M. Heller-G. Roucaute (c) Inventaire général, ADAGP, 1998.

Pontremoli dut résoudre une toute autre difficulté pour répondre à la demande de Fanny Reinach qui tenait absolument à disposer d'un piano. Celui-ci présente l'aspect d'une armoire à deux corps dont rien ne laisse supposer qu'il s'agit d'un instrument de musique. Il faut en ouvrir les battants qui se replient entièrement, abaisser et faire basculer le clavier, relever la partie supérieure et, enfin, déplier le porte-partitions pour pouvoir jouer (fig. $\mathbf{n}^{\circ}$ 9) (fig. $\left.\mathbf{n}^{\circ} \mathbf{1 0}\right)$. Même les pédales sont masquées par un petit élément en forme de coffret. 
Figure 9

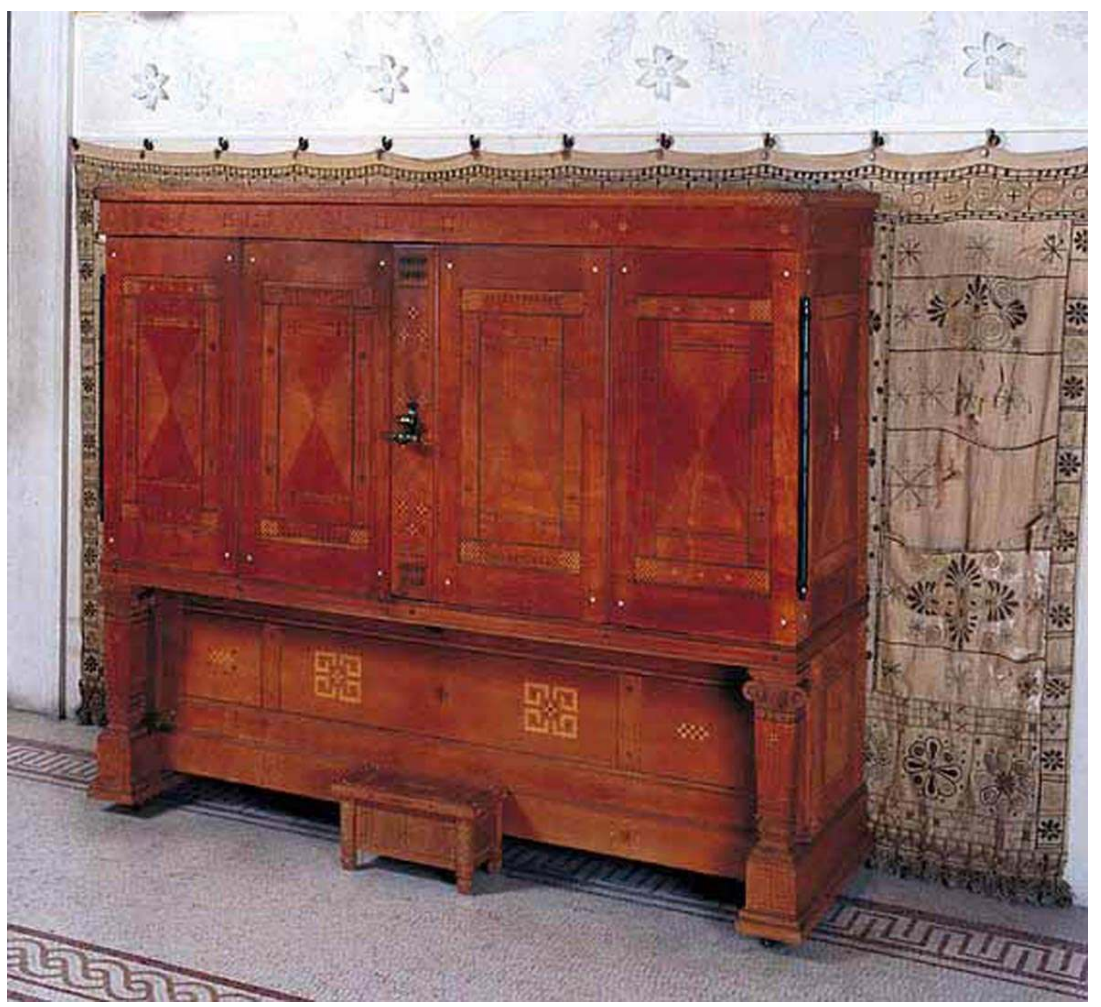

Le piano, fermé.

Phot. Inv. M. Heller-G. Roucaute (c) Inventaire général, ADAGP, 1998. 
Figure 10

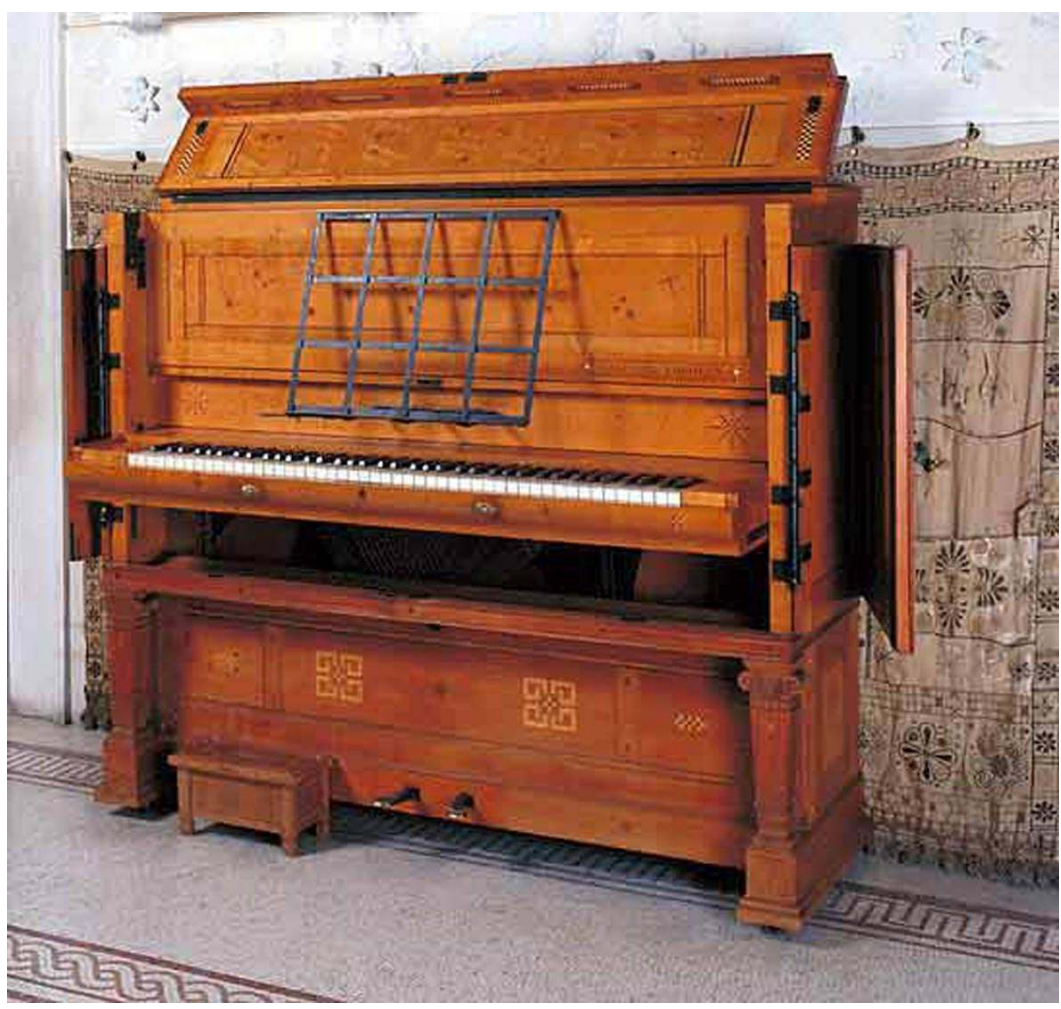

Le piano, prêt à être joué.

Phot. Inv. M. Heller-G. Roucaute @ Inventaire général, ADAGP, 1998.

Ce souci du détail, l'ingéniosité déployée pour associer confort et vérité archéologique révèlent le talent de Pontremoli qui a su trouver d'habiles solutions tant pour le mobilier que pour l'architecture.

\section{L'originalité des matériaux et la qualité de leur mise en œuvre}

14 La recherche de matériaux rares, la perfection de la mise en œuvre et le raffinement du décor d'incrustations accentuent le caractère exceptionnel de ce mobilier. L'identification des bois, dont certains sont d'un usage peu courant, est possible grâce aux dessins préparatoires de Pontremoli, conservés dans les archives de l'Institut de France, qui constituent l'essentiel des sources disponibles sur la villa ${ }^{5}$, les archives de la famille Reinach ayant été dispersées, sinon détruites, lors de la seconde Guerre mondiale. Ces croquis sur papier-calque indiquent non seulement les formes et les cotes des meubles, mais aussi tous les décors en précisant la nature des bois à travailler.

15 La plupart sont des bois exotiques, aux noms très poétiques, comme le vert de Siam, le prunier d'Australie, le cocobolo, le noyer d'Amérique ou le citronnier de Ceylan. Parmi les bois indigènes, le houx est fréquemment employé en toutes petites incrustations ; le hêtre est plutôt utilisé pour le décor, malgré son caractère non précieux. Remarquons aussi que si une essence existe parmi les bois indigènes, c'est sa variante exotique qui est 
recherchée: noyer d'Amérique, olivier sauvage de Cochinchine, prunier d'Australie, citronnier de Ceylan.

Les projets révèlent également qu'à chaque pièce correspondait un ensemble de meubles, réalisé avec le même bois de bâti (souvent traité en placage) et un décor d'incrustations associant de deux à six bois différents ${ }^{6}$. Les bois composant les structures sont surtout l'angélique, le noyer d'Amérique, le citronnier, le « vert de Siam », le « cœur de vert » et le palissandre mais ils sont tous également utilisés pour les incrustations. Les seuls matériaux employés uniquement pour le décor sont l'ivoire, l'ébène, le houx, l'acajou et, très rarement, la nacre.

Les projets de deux ensembles importants, destinés à la bibliothèque et au petit salon, ne semblent pas avoir été conservés et nous avons tenté d'en identifier les matériaux en les comparant avec ceux notés par Pontremoli sur les autres projets. Il fallut tout d'abord rapprocher les dessins des meubles, ce qui permit d'observer un certain nombre de différences, surtout des inversions entre les bois de structure et ceux de décor ou entre deux bois de décor, voire un changement complet de matériau, ce qui est le cas pour un assez grand nombre de tabourets ${ }^{7}$. Le poirier prévu pour une des chambres du second étage a été remplacé par du citronnier, sauf pour une table. Le chêne, pourtant largement présent, n'est pas cité par Pontremoli, mais il est vrai qu'il constitue surtout le dos ou l'intérieur des meubles.

Les modifications constatées nous incitèrent à vérifier si les bois exotiques que nous ne connaissions pas étaient bien ceux indiqués sur les croquis. Après consultation d'un spécialiste ${ }^{8}$, il apparut que le cocobolo a été remplacé par un bois plus léger, plus clair et aux pores plus larges, qui n'est pas actuellement identifié. Pontremoli distingue le « vert de Siam » du " cœur de vert », dont les différences sont peu visibles à l'œil nu. Le premier de ces matériaux a pu être reconnu comme du cœur vert, bois très dense, devenant rougeâtre en vieillissant et nous avons supposé que le « cœur de vert » en était la partie centrale. Quant au « corail », il s'agit en réalité d'un bois exotique, le padouk, et non de la matière calcaire semi-précieuse. Tous les autres matériaux concordent avec les projets.

L'ébéniste a scrupuleusement respecté les formes des meubles et des incrustations prévues par l'architecte-décorateur et il est probable qu'il ait rencontré des difficultés pour se procurer tous les bois demandés ou pour les travailler. Il subsiste donc des incertitudes en ce qui concerne certaines incrustations des meubles de la bibliothèque et $\mathrm{du}$ petit salon, à l'exception de celles du piano. Dans celui-ci, réalisé a posteriori, sont associés les motifs et les bois de deux ensembles différents, la chambre de Madame et le salon de repos du premier étage, auxquels s'ajoutent des incrustations analogues à celles des grandes tables de la bibliothèque. 


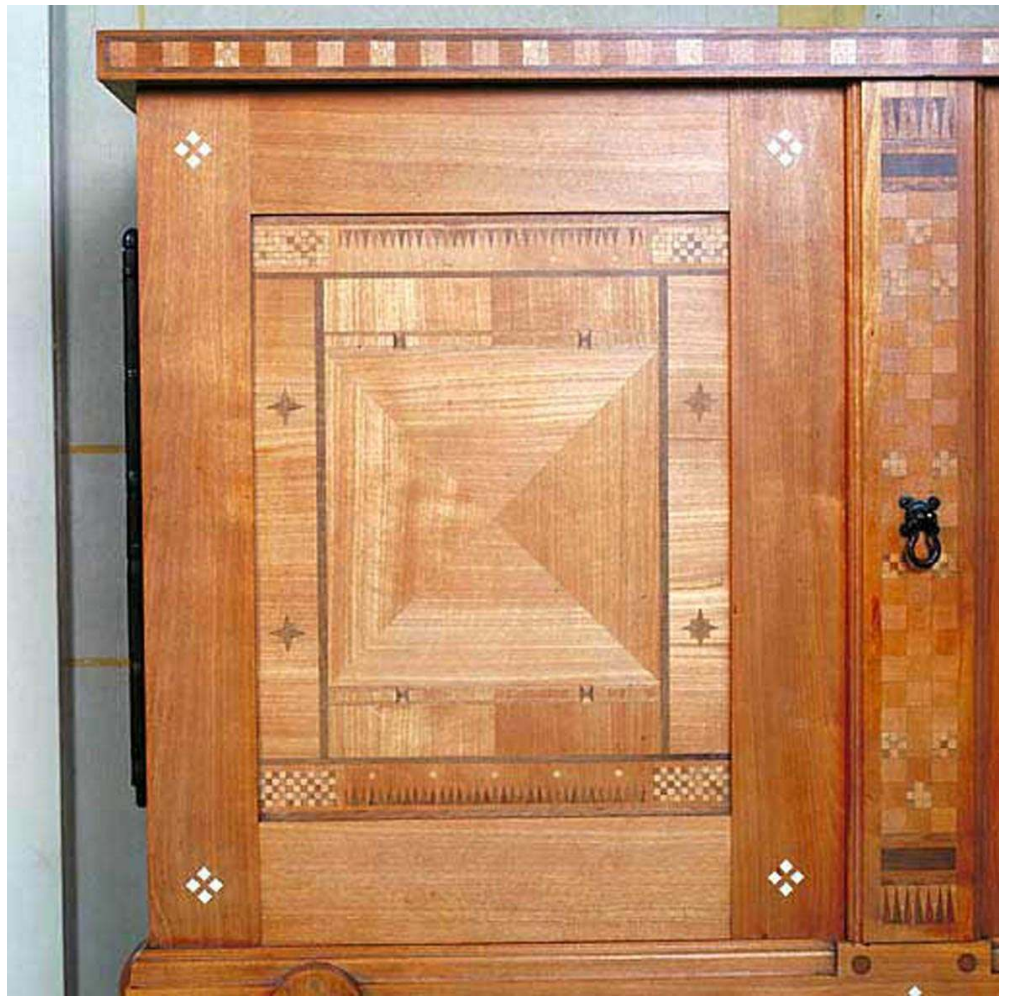

Le décor d'une armoire de la chambre de Madame, en citronnier incrusté de noyer d'Amérique, d'olivier et de houx

Phot. Inv. M. Heller-G. Roucaute @ Inventaire général, ADAGP, 1998.

La qualité des matériaux est mise en valeur par une facture quasi parfaite tant pour les assemblages et les placages que pour les incrustations, exécutés sur ces dessins par les ateliers de Louis-François Bettenfeld. Soulignons en particulier le procédé décoratif consistant à plaquer les grandes surfaces de triangles ou de carrés découpés dans la même feuille, en opposant le sens du fil, afin de jouer avec la lumière (fig. $\left.\mathbf{n}^{\circ} \mathbf{1 1}\right)$. Il est fréquent que des incrustations soient réalisées avec le bois utilisé pour la structure mais taillé ou placé dans un sens différent, donnant ainsi l'illusion d'un matériau supplémentaire. Les parties cachées des meubles sont traitées avec autant de soin que les surfaces visibles. Par exemple, le dessous des plateaux des grandes tables de la bibliothèque est plaqué exactement de la même façon que le dessus avec des triangles d'angélique disposés en sens contraire dans un encadrement de prunier d'Australie et de citronnier (fig. $\mathbf{n}^{\circ} \mathbf{1 2}$ ). Ce procédé est fréquemment employé pour l'intérieur des battants. Le raffinement est poussé à l'extrême car même l'intérieur du coffre à linge de la salle de bains de Madame, qui n'est pourtant pas destiné à être vu, est plaqué de carrés de chêne taillés de façon à ce que les veines soient placées à angle droit. (fig. $\mathrm{n}^{\circ} \mathbf{1 2}$ ) 


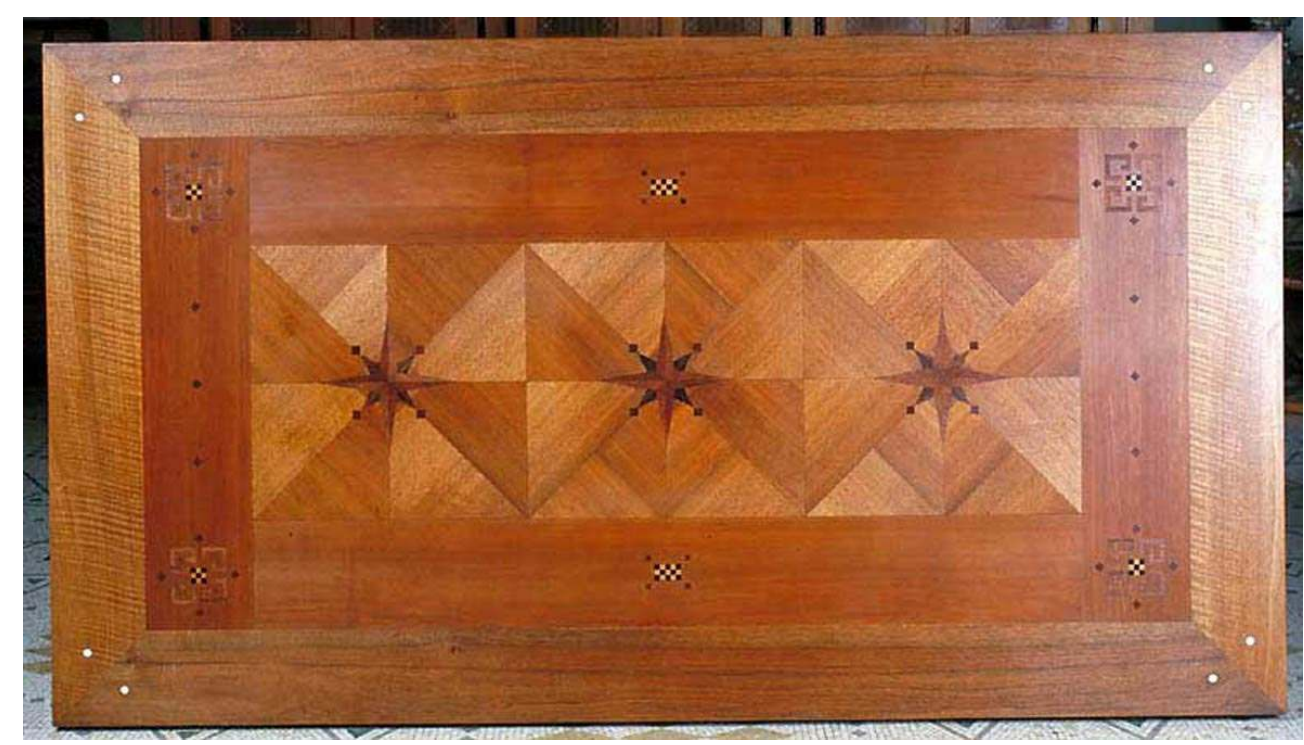

Le dessus des grandes tables de la bibliothèque, plaqué de triangles d'angélique de sens contraire, dans un encadrement de prunier d'Australie ; les traverses du plateau sont en citronnier.

Phot. Inv. M. Heller-G. Roucaute ( $)$ Inventaire général, ADAGP, 1998.

21 L'emploi quasi systématique de bois exotiques, qui pourrait paraître s'inscrire dans un courant à la mode, relève lui aussi du souci de vérité archéologique. En effet, dans les riches demeures grecques, les meubles et surtout les lits, pièces d'apparat, étaient confectionnés dans des bois très variés, indigènes et exotiques, et la recherche décorative était très poussée. Les placages et les incrustations étaient fréquents, avec une préférence pour le houx, l'ébène et la loupe de thuya. Un raffinement supplémentaire consistait à plaquer des lames d'écailles de tortue afin d'imiter les bois précieux. Le cœur vert, avec son aspect rougeâtre, a peut-être été choisi pour remplacer le pistachier de Syrie très prisé dans l'Antiquité'.

\section{Les formes et le décor : une uniformité apparente, une variété dans le détail}

Un examen attentif révèle que les structures et les formes peu variées en apparence comportent en réalité de nombreuses et subtiles différences. La plupart des tables ont trois pieds, mais ceux-ci ne sont jamais identiques même s'ils s'achèvent souvent en patte de lion plus ou moins stylisée. Certains pieds sont gainés de bronze, ils sont parfois reliés par des traverses d'entretoise et leur assemblage avec le plateau est de deux types. Les chevilles en ivoire, en réalité des pastilles incrustées, sont rondes ou carrées, ces dernières pouvant être groupées par quatre. Les garnitures des sièges sont pratiquement toutes composées de lanières de cuir mais celles-ci sont rondes et étroites, ou plates et de diverses largeurs; elles sont tressées une à une ou par groupe de trois; soit elles passent sur la ceinture et sont clouées au-dessous ou à l'intérieur, soit elles entourent un cadre lui-même encastré dans la ceinture. Les très nombreux tabourets comportent au moins une dizaine de formes différentes contrairement à ceux présentés sur les vases antiques. Il existe seulement quatre modèles de chaises, qui présentent parfois les mêmes motifs décoratifs, tout en étant dissemblables grâce aux bois employés. 
Le décor des meubles, des mosaïques, des fresques, des tentures et de la vaisselle peut paraître répétitif car il est constitué à partir d'un petit nombre d'éléments de base. La juxtaposition de carrés, de triangles et de disques génère surtout des damiers, des étoiles et des rosaces qui sont associés à des formes plus élaborées de volutes et de palmettes (fig. $\mathbf{n}^{\circ}$ 13). Mais les multiples combinaisons produisent un résultat toujours varié.

\section{Figure 13}

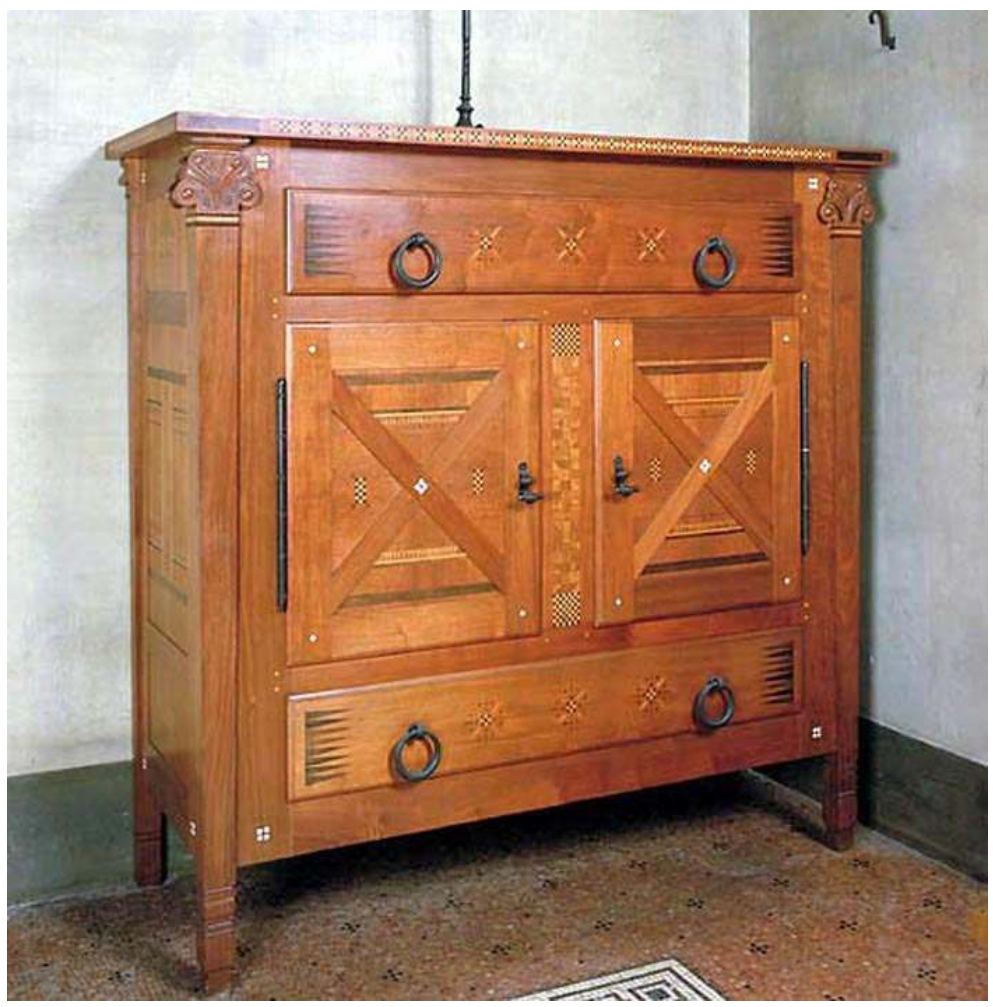

L'armoire de la chambre de Monsieur, en noyer d'Amérique.

Phot. Inv. M. Heller-G. Roucaute (c) Inventaire général, ADAGP, 1998.

24 L'ornementation la plus complexe est celle des buffets en cœur vert du grand salon, dont les battants et les panneaux des côtés sont entièrement incrustés d'olivier, de citronnier, de palissandre, de pseudo-cocobolo et de houx, ainsi que de petits triangles de cœur vert (fig. $\mathbf{n}^{\circ}$ 14). La réalisation de ce projet comporte le plus grand nombre de modifications, l'alternance de bois clair et de bois foncé ayant été inversée. Les incrustations qui auraient dû être en citronnier ont été faites en cœur vert et vice-versa, celles en palissandre sont en pseudo-cocobolo. 
Figure 14

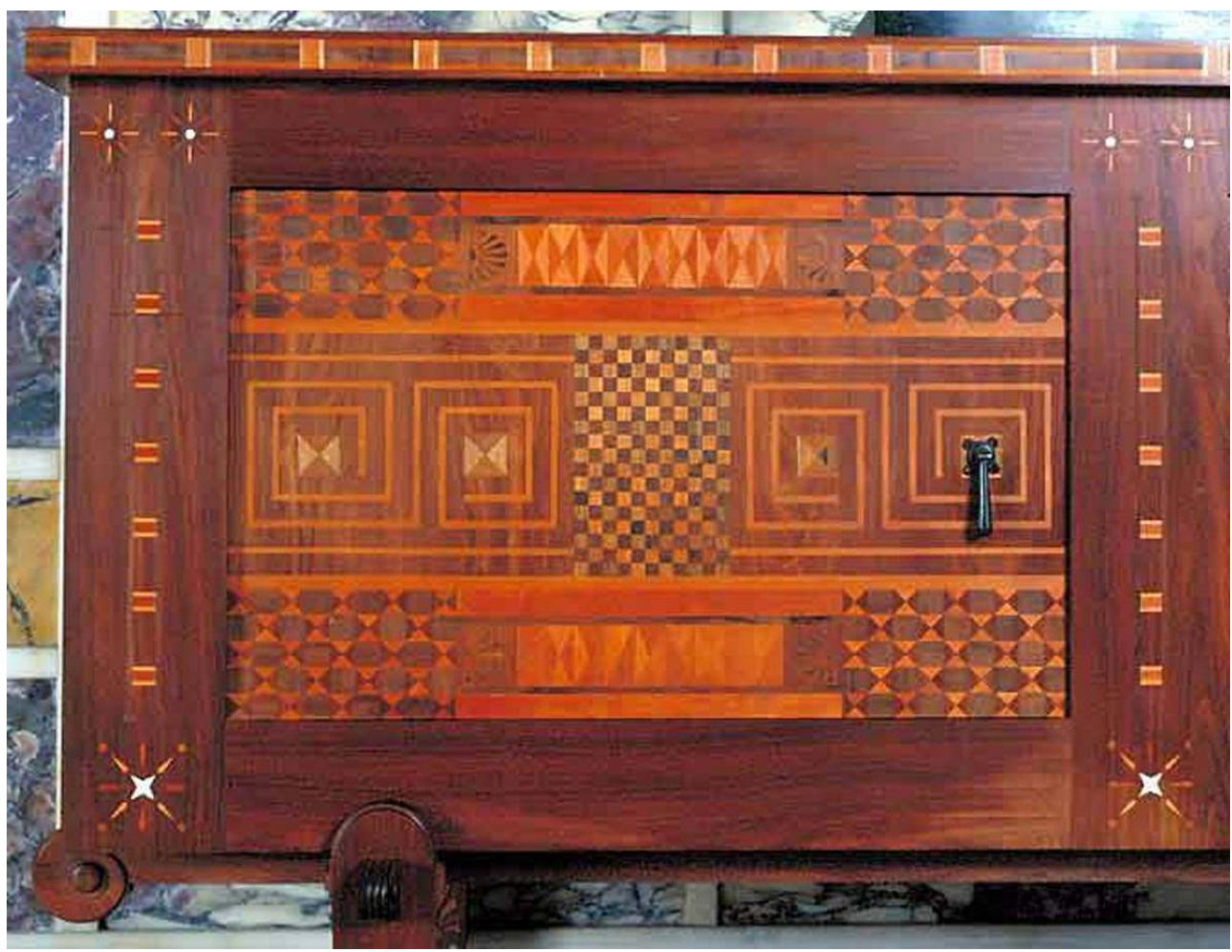

Le décor d'un buffet du grand salon, en cœur vert incrusté de cinq autres bois.

Phot. Inv. M. Heller-G. Roucaute (c) Inventaire général, ADAGP, 1998.

Même dans le décor le plus simple (fig. $\mathbf{n}^{\circ}$ 15), celui de la chambre d'amis du premier étage (étoiles et palmettes, rangées de triangles, quelques damiers sur le plateau de la table), l'observation révèle qu'il y a trois types d'étoiles et que toutes les volutes et les palmettes sont différentes. Remarquons aussi que le thème décoratif de chaque ensemble mobilier n'est pas semblable à celui de la décoration murale de la pièce dans laquelle il se trouve mais est assorti à celui d'une autre pièce, créant ainsi une grande harmonie dans la demeure. 
Figure 15

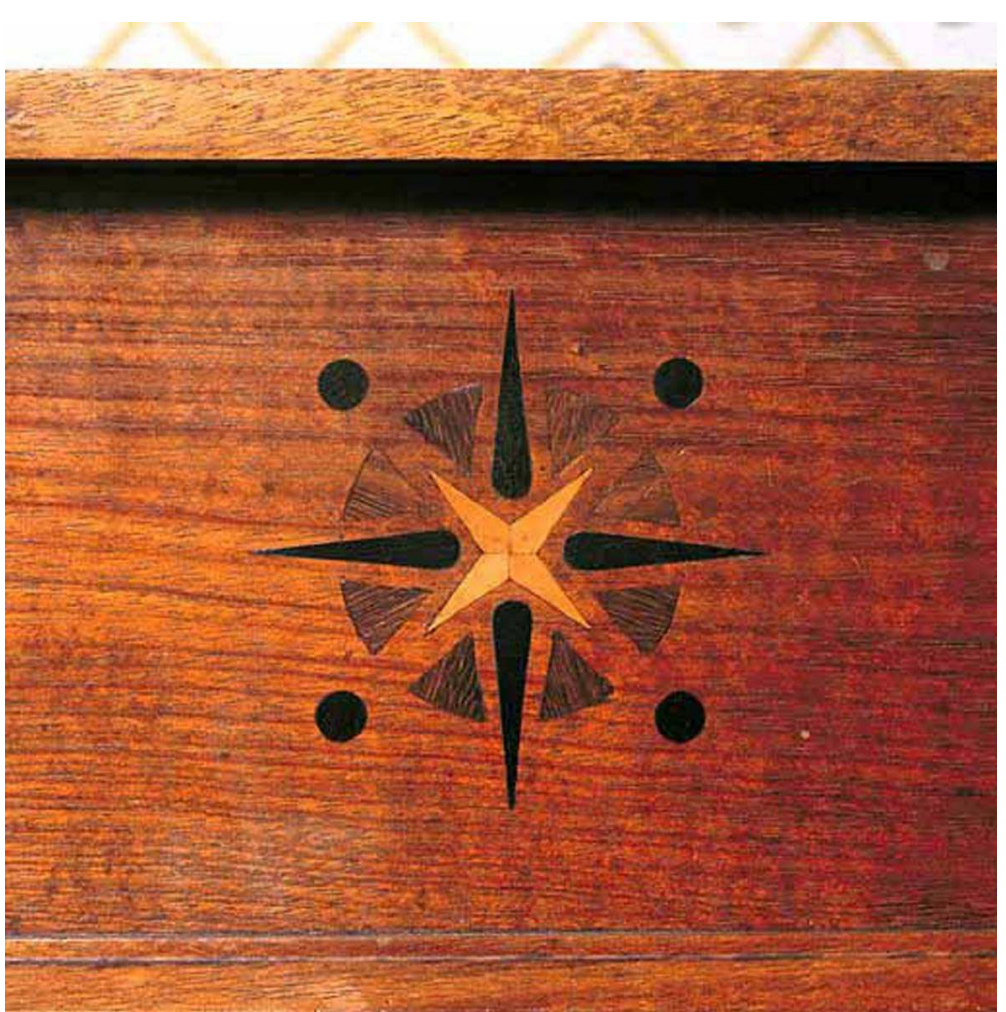

Le détail des incrustations en houx, ébène et « corail » de la toilette de la chambre d'amis du premier étage.

Phot. Inv. M. Heller-G. Roucaute (c) Inventaire général, ADAGP, 1998.

L'importance attachée à la notion d'ensemble et à la destination spécifique de chaque œuvre est révélée par les annotations de Pontremoli sur ses dessin, par exemple : « deux chaises pour la chambre angélique, chambre d'amis du $1^{\text {er }}$ étage ", qui complète l'indication des matériaux, "corail», ébène et houx (fig. $\left.\mathbf{n}^{\circ} \mathbf{1 6}\right)$. Ce projet est le seul conservé pour la totalité de l'ameublement de la pièce, dont une bonne partie n'est plus en place à commencer par les deux chaises ${ }^{10}$. Sans lui, il aurait été impossible d'identifier et de réunir tous les éléments de l'ensemble dont les motifs décoratifs ne sont pas identiques. 


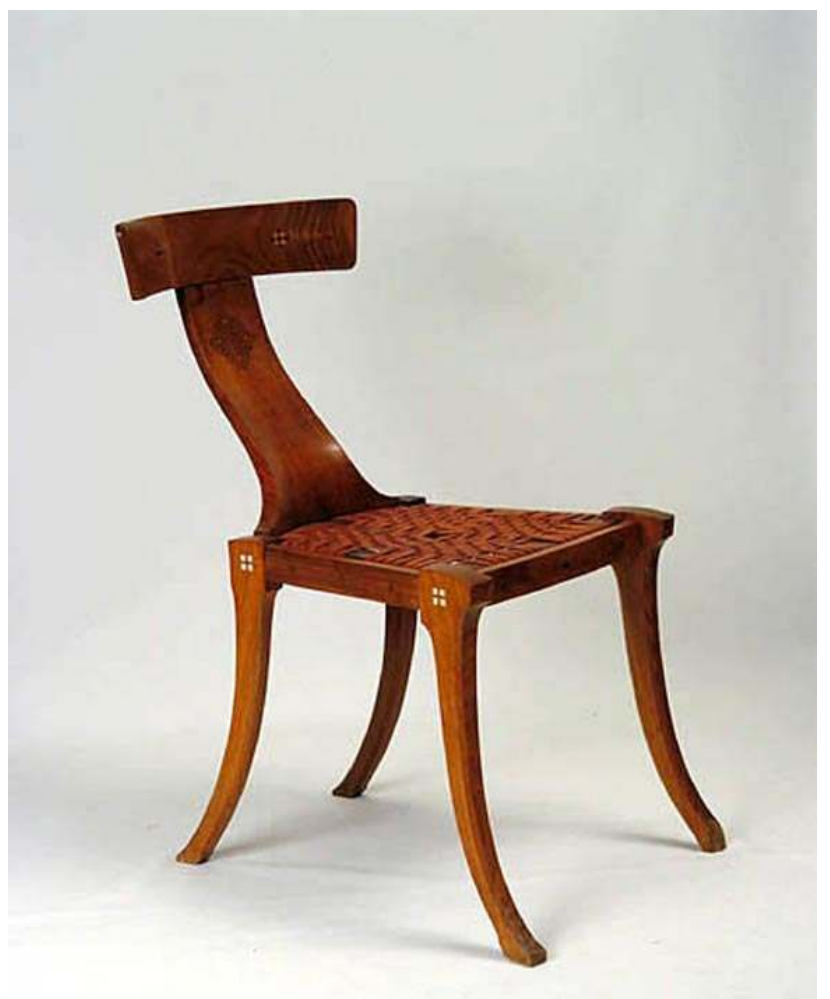

La chaise de la chambre d'amis du premier étage.

Phot. Inv. M. Heller-G. Roucaute (c) Inventaire général, ADAGP, 1998.

Pourquoi accorder autant d'intérêt à cette reconstitution des ensembles? C'est qu'elle constitue la première étape de toute étude faite suivant la méthodologie de l'Inventaire général, surtout dans le domaine du mobilier, et qu'elle est indispensable à la compréhension des œuvres. Dans la villa Kérylos, la tâche fut rendue particulièrement délicate par l'uniformité apparente des formes et des décors qui empêche de voir immédiatement à quel ensemble appartient un meuble qui n'est plus en place. Or, tous les ensembles mobiliers ne sont plus totalement homogènes, quand ils n'ont pas été entièrement dispersés, comme ceux des chambres d'amis du rez-de-chaussée et du deuxième étage. La plupart des petits coffres agrémentent les couloirs et les paliers d'escalier et un tiers des sièges ne se trouve plus dans sa pièce d'origine. Seule une couverture photographique presque complète, associée à l'analyse des dessins de Pontremoli, nous a permis de mener à bien cette étape préliminaire. 


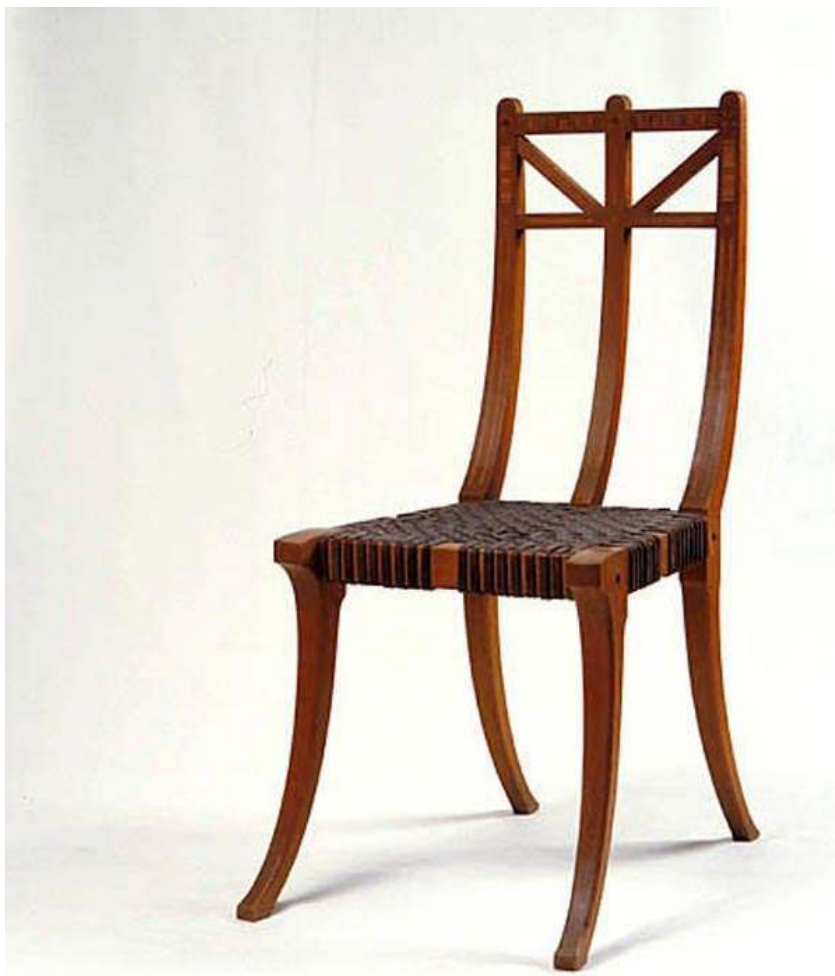

Une « chaise légère », destinée à la salle de bains de Monsieur.

Phot. Inv. M. Heller-G. Roucaute (c) Inventaire général, ADAGP, 1998.

$\mathrm{Au}$ cours de la recherche documentaire, nous avons constaté de grandes similitudes entre les copies qui complètent le mobilier réalisé par Bettenfeld et les œuvres illustrant le Dictionnaire des antiquités grecques et romaines de Daremberg, Saglio et Pottier, publié à partir de 1877, comme le lit en bronze de la chambre de Monsieur et diverses petites tables à piétement en bronze, répliques de meubles découverts à Pompéi. La composition du dossier des "chaises légères ", avec trois montants fins reliés par de petites traverses, pourrait également trouver sa source dans cet ouvrage (fig. $\mathbf{n}^{\circ} \mathbf{1 7}$ ); il en est de même pour les lampes suspendues en terre cuite, en forme de couronne à plusieurs becs ${ }^{11}$. Enfin, bien que cela nous éloigne un peu du sujet, nous tenons à signaler que les formes et les décors des couverts en argent, assez hétéroclites, sont exactement les mêmes que ceux des objets présentés dans le Dictionnaire des antiquités. Certains manches se terminent en sabot de biche comme celui de la cuillère trouvée en Asie Mineure, d'autres s'achèvent en chapelet de boules comme celui d'une cuillère romaine découverte à Canterbury ${ }^{12}$. Quant aux petites cuillères au manche fin et pointu, elles ressemblent étrangement à l'illustration de la rubrique cochlear $^{13}$ (fig. $\mathbf{n}^{\circ} \mathbf{1 8}$ ). Il n'est cependant pas possible d'affirmer qu'il s'agit simplement de copies de ces planches, Pontremoli et Reinach ayant suffisamment de relations avec le milieu de l'archéologie pour connaître les rapports de fouilles qui ont alimenté l'ouvrage. Toutefois, les différentes pièces du mobilier sont fonctionnelles tout en étant de véritables œuvres d'art et jouent pleinement leur rôle de témoins de l'Antiquité. 


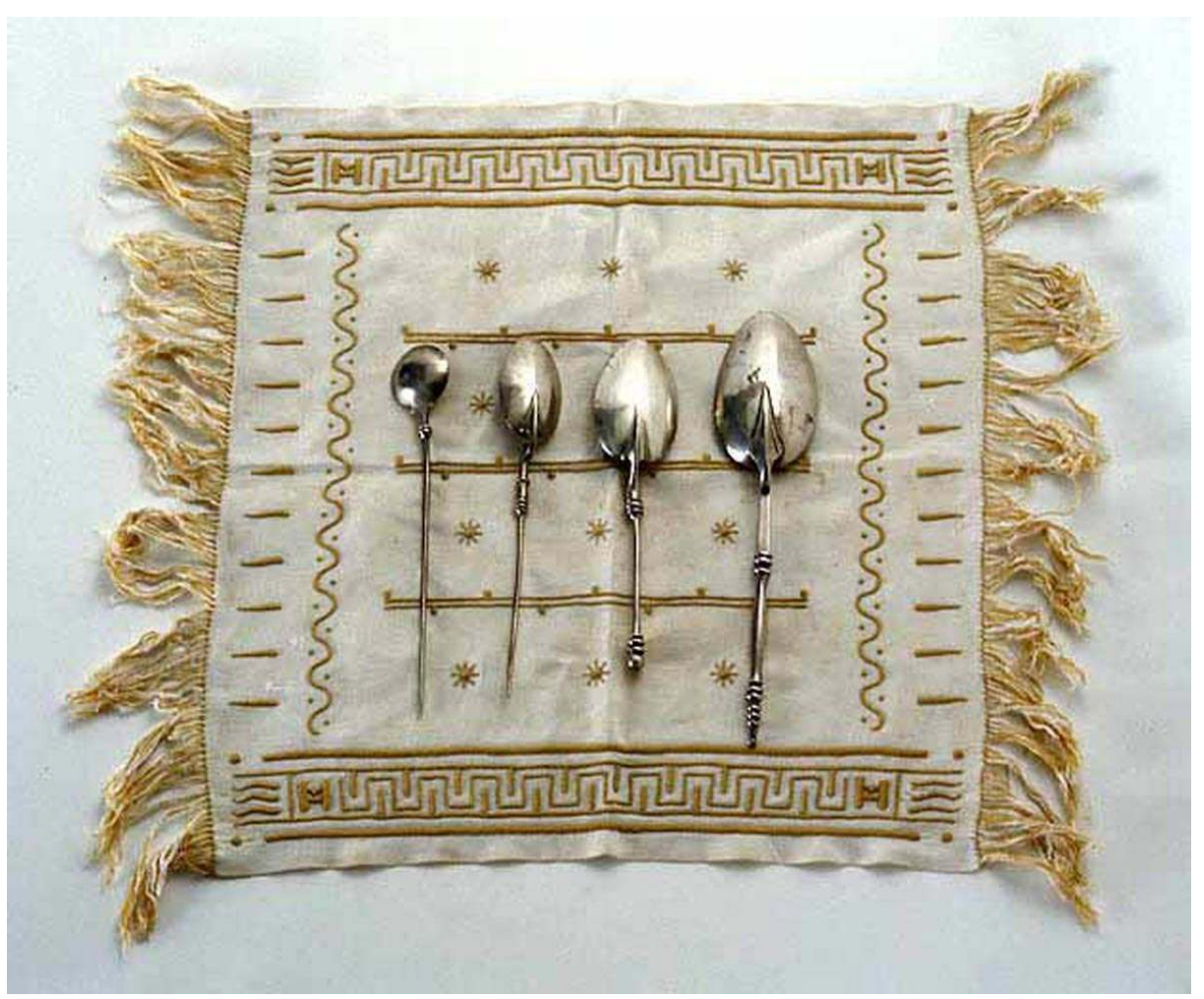

Les différentes formes de cuillères en argent, copiées sur des modèles antiques et réalisées par l'orfèvre parisien Leverrier et Cie.

Phot. Inv. M. Heller-G. Roucaute (c) Inventaire général, ADAGP, 1998.

Les meubles de la villa Kérylos s'intègrent si parfaitement dans leur cadre architectural que l'on tend à oublier le caractère exceptionnel de leur conception et de leur réalisation. Ils ont été imaginés par Pontremoli après une analyse minutieuse de la seule source antique disponible, la peinture céramique ou murale, peut-être complétée par l'étude de récentes publications. La qualité de l'exécution, sans laquelle la création n'aurait pu être aboutie, doit également être soulignée. Le travail $\mathrm{du}$ bois et la perfection des incrustations, pourtant faites de très petits éléments, révèlent la maitrise des artisans qui les ont réalisés sous la direction de Louis-François Bettenfeld. Les meubles se trouvent depuis près d'un siècle dans une maison au bord de la mer, mais ni les assemblages ni les incrustations n'ont joué et les dégradations sont seulement accidentelles ${ }^{14}$. Enfin, au-delà du pari archéologique, rappelons la gageure technique que représentait l'introduction du confort moderne et sa dissimulation dans une maison à l'antique. Pontremoli sut résoudre tous les problèmes de manière habile et élégante, tant pour l'architecture que pour le mobilier.

Contrairement au décor, qui inspira celui de la Villa Primavera à Cap-d'Ail, l'ameublement de la villa Kérylos reste un unicum, qui ne s'insère dans aucun courant artistique. Il doit sa naissance à la conjonction de deux génies créateurs, qui eurent la chance et les moyens de trouver des artistes et des artisans à la hauteur de leur projet extraordinaire ${ }^{15}$. 
NOTES

1. ${ }^{1}$ Nous ne reviendrons pas sur les biographies de ces deux personnages, qui sont développées par M. Vian des Rives dans un chapitre de l'intéressant ouvrage collectif La Villa Kérylos, publié en 1997 aux Editions de l'Amateur. Elles complètent les notices présentées dans : CHAMONARD, J., PONTREMOLI, E. Kérylos, la villa grecque. Paris, 1934, réédité en 1996 par Jeanne Laffitte. Voir aussi, sur le site du ministère de la Culture: http://www.culture.gouv.fr/documentation/ merimee/accueil.htm

http://www.culture.gouv.fr/documentation/palissy/accueil.htm (interroger : Beaulieu-sur-Mer). 2. Les cinq cents pièces de vaisselle, créées par Emile Lenoble entre 1909 et 1911, l'argenterie de la maison parisienne Leverrier et Cie et la verrerie furent également réalisées suivant ses directives, dans le même souci de reconstitution archéologique.

3. Cet artisan peu connu est certainement Louis-François Bettenfeld, fils de l'ébéniste François Bettenfeld et d'Agnès Keller, né le 5 juin 1855 à Paris. Selon le Bottin de commerce, il était installé dans le Faubourg Saint-Antoine depuis 1891 et, en 1905, avait trois adresses voisines dans le même quartier. En 1920, il travaillait encore, avec ses fils. La mention ébéniste disparaît avant 1925, date à laquelle la recherche s'est limitée.

4. CHAMONARD, J., PONTREMOLI, E. Kérylos, la villa grecque. Paris, 1934, réédité en 1996 par Jeanne Laffitte. La planche XVI présente ce mobilier en place.

5. Nous avons consulté environ 90 dessins, presque tous numérotés, concernant environ 130 meubles ou objets étudiés, sans avoir la certitude qu'il n'en existe pas davantage, le fonds n'étant pas classé.

6. Dans les précédentes publications, les auteurs indiquent le plus souvent « du bois incrusté » et précisent seulement que les meubles du petit salon sont en citronnier. Chamonard, le premier, cite seulement quatre essences (chêne, citronnier, bois de violette et camphrier), tout en commettant une erreur en ce qui concerne les grandes tables de la bibliothèque dont le bâti apparent n'est pas en chêne mais en citronnier et en angélique. Quant au camphrier, il est présent sur un seul meuble.

7. Par exemple, le projet pour deux tabourets de forme identique, l'un en «corail » incrusté de « cocobolo » et de palissandre, l'autre en « cocobolo » incrusté de «corail » et de palissandre, a donné lieu à la réalisation de trois sièges, l'un en palissandre, l'autre en chêne teinté palissandre et le troisième en chêne clair, tous incrustés de houx et de palissandre.

8. L'identification des bois exotiques rares a pu être faite grâce à M. Patrick George, que nous ne saurions assez remercier pour son obligeance et la grande disponibilité dont il a fait preuve. Précisons cependant qu'il ne lui a pas été possible de se rendre sur place et que nous avons travaillé sur des photographies et sur nos propres observations et que, par conséquent, toutes les questions n'ont pu être résolues. Il semble que le cocobolo ait été remplacé, mais nous ignorons par quel bois. De même, nous n'avons pas vérifié que l'ébène était véritable.

9. Dictionnaire des antiquités grecques et romaines, d'après les textes et les monuments... sous la direction de MM. Ch. Daremberg, E. Saglio et E. Pottier. Paris : Librairie Hachette, tome III, 1904, article materia.

10. Les chaises sont actuellement dans la salle à manger, la table de nuit dans le salon de repos du premier étage, les marchepieds des lits dans le grand salon, deux coffres et deux tabourets au deuxième étage. 
11. Dictionnaire des antiquités grecques et romaines, d'après les textes et les monuments.. sous la direction de MM. Ch. Daremberg, E. Saglio et E. Pottier. Paris : Librairie Hachette, tome III, 1904, p. 1324, fig. 4579.

12. Dictionnaire des antiquités grecques et romaines, d'après les textes et les monuments.. sous la direction de MM. Ch. Daremberg, E. Saglio et E. Pottier. Paris : Librairie Hachette, tome III, 1904, fig. 4485 et 4487.

13. Dictionnaire des antiquités grecques et romaines, d'après les textes et les monuments... sous la direction de MM. Ch. Daremberg, E. Saglio et E. Pottier. Paris : Librairie Hachette, tome I, 1904, p. 1266, fig. 1689.

14. Il faut toutefois préciser que de nombreux sièges en cœur vert sont cassés, ce bois étant très lourd et inadapté aux formes très incurvées et aux porte-à-faux.

15. La villa aurait coûté neuf millions de francs-or. La villa Kérylos, 1997, p. 27.

\section{RÉSUMÉS}

La villa Kérylos fut construite à Beaulieu-sur-Mer entre 1902 et 1908, par Emmanuel Pontremoli pour l'helléniste Théodore Reinach, à la manière d'une maison antique. L'architecte fut également le concepteur de l'intégralité du décor et du mobilier, inspirés par l'Antiquité grecque et romaine, y compris les objets usuels, comme la vaisselle fabriquée par Emile Lenoble et l'argenterie de la maison parisienne Leverrier et Cie. Les meubles, pour la plupart en bois exotiques décorés d'incrustations, ont été réalisés par l'ébéniste Louis-François Bettenfeld, à partir de dessins précis de Pontremoli. L'analyse des croquis et l'étude des œuvres a permis d'identifier les bois et de reconstituer les ensembles prévus par l'architecte pour chaque pièce. La combinaison d'un petit nombre de motifs de base produit une grande variété de décors tout en créant une grande harmonie dans la demeure car les thèmes décoratifs se répondent d'une pièce à l'autre. La qualité d'exécution des meubles, dont les formes se rapprochent le plus possible des modèles antiques visibles sur les céramiques figurées, est telle que l'on peut les qualifier de chefs-d'œuvre d'ébénisterie. Il faut enfin souligner la prouesse technique consistant à intégrer et à dissimuler parfaitement les commodités modernes, électricité, eau courante et chauffage central, dans un cadre « antique».

The Kérylos villa was built at Beaulieu-sur-Mer between 1902 and 1908 to the designs of the architect Emmanuel Pontremoli for the Hellenist Théodore Reinach. The villa was conceived as a house of classical antiquity. The architect was also responsible for all the villa's interior design and furniture, similarly inspired by Greek and Roman antiquity. Even everyday object like the tableware, made by Emile Lenoble, and the silverware, coming from the Paris manufacturer Leverrier et Cie, was of classical inspiration. Most of the furniture is of wood and was executed by the cabinet-maker Louis-François Bettenfeld, following detailed designs by Pontremoli. The analysis of the architect's sketches and the study of the actual pieces of furniture has allowed for the different types of wood to be identified and for a better understanding of the architect's intentions for each of the villa's rooms. The combination of a relatively limited number of basic decorative motifs produces a broad variety in the decors and an overall harmony throughout the villa, the decorative themes echoing one another from one room to the next. The forms of the different items of furniture are as close as possible to models found on classical vase paintings, and the quality of their execution allows them to be described as cabinet-making masterpieces. It 
is also worth drawing attention to the technical prowess in the perfect dissimulation of features of modern comfort (electricity, hot water, central heating) in this "antique" environment.

\section{INDEX}

Keywords : villa, Kérylos, Beaulieu-sur-Mer, Théodore Reinach, Fanny Reinach, Pontremoli, Bettenfeld, antiquité grecque, néo-grec, bois exotique, meuble, mobilier, antique, ébénisterie, piano, ornement, décor, incrustation, bois exotique, argenterie, Leverrier, Emile Lenoble, Délos, Côte d'Azur, villégiature, coffre, chaise, piano, incrustation, marqueterie, bois, noyer d'Amérique, citronnier, olivier de Cochinchine, prunier d'Australie, citronnier de Ceylan, ivoire, ébène, houx, acajou, nacre, cobocolo, padouk, décor, décorateur, thuya, écaille de tortue, pistachier de Syrie, tabouret

\section{AUTEUR}

\section{FRANÇOISE REYNIER}

Ingénieur d'études. Inventaire. Région Provence-Alpes-Côte d'Azur.

francoise.reynier@culture.gouv.fr 